\title{
Tryptophan-Kynurenine Metabolites in Exercise and Mental Health
}

\author{
Paula Valente-Silva and Jorge Lira Ruas
}

\begin{abstract}
In our efforts to identify molecular mediators of the benefits of exercise to human health, we have uncovered a biochemical pathway in skeletal muscle that positively impacts mental health. This mechanism is activated by endurance training and controlled by the transcriptional coactivator PGC- $1 \alpha 1$, which induces transcription of several kynurenine aminotransferase (KAT) genes in muscle. KAT enzymes catabolize the neuroinflammatory tryptophan metabolite kynurenine, which can accumulate in the brain and lead to alterations associated with stressinduced depression (among other psychiatric diseases). Here, we discuss our findings in the context of what is known about the kynurenine pathway of tryptophan degradation and how its many metabolites can directly affect the brain. These findings provide a mechanism for how physical exercise can improve mental health and offers potential therapeutic targets for future antidepressant medications.
\end{abstract}

\section{Introduction}

The many benefits of physical exercise to human health are widely recognized. Depending on their type, intensity, duration, and frequency, different exercise modalities can be used to improve diverse physiological parameters (Hawley et al. 2014). These include cardiovascular fitness, strength, energy metabolism, and resistance to fatigue, among others. Importantly, exercise training can be used as a prophylactic or therapeutic intervention for a variety of pathologies ranging from obesity and diabetes to cancer and mental health disorders (Cooper et al. 2017; Hawley 2004). Indeed, maintaining an active lifestyle continues to be the best way to promote healthy longevity. However, the molecular mechanisms that allow the human body to adapt to exercise challenges are still poorly understood. This is true

P. Valente-Silva • J. L. Ruas $(\bowtie)$

Department of Physiology and Pharmacology, Molecular and Cellular Exercise Physiology,

Karolinska Institutet, Stockholm, Sweden

e-mail: jorge.ruas@ki.se

B. Spiegelman (ed.), Hormones, Metabolism and the Benefits of Exercise,

Research and Perspectives in Endocrine Interactions,

https://doi.org/10.1007/978-3-319-72790-5_7 
for individual tissues and organs but even more so for the network of inter-organ communication events that coordinates whole-body adaptation to the multitude of stimuli that exercise training entails. Skeletal muscle plays a central role in this process and has been the most studied tissue in this context. From this work, we have learned valuable information about key players in the regulation of muscle function, such as the peroxisome proliferator-activated receptor (PPAR)- $\gamma$ coactivator- $1 \alpha$ (PGC-1 $\alpha$ ) proteins (Correia et al. 2015). This still expanding family of transcriptional coactivators is composed of several splicing variants with different biological activities and discrete regulation, important for the many effects of physical exercise (Martínez-Redondo et al. 2015). By understanding these mechanisms, we have also started to elucidate how myokines (muscle-derived factors with local and/or distal effects) communicate to the rest of the body the changes in skeletal muscle elicited by exercise (Giudice and Taylor 2017). For example, exercise training can reduce the levels of several mediators of chronic low-grade inflammation (Handschin and Spiegelman 2008), which increase with sedentary habits. This kind of sustained, unresolved, low-grade sterile inflammation has been linked to the etiology of many diseases such as diabetes, cancer, and depression. In this context, the discovery that trained muscle can actively participate in the catabolism of neurotoxic tryptophan metabolites with known deleterious effects on mental health (Agudelo et al. 2014) has added another layer of complexity to the many functions of exercised muscle.

\section{The PGC-1 $\alpha$ Family of Transcriptional Coactivators in Skeletal Muscle}

Although our understanding of the mechanisms that regulate skeletal muscle adaptation to different exercise challenges remains incomplete, PGC- $1 \alpha$ coactivators have been shown to play important roles in this process. These proteins are expressed in energy-demanding tissues such as heart, skeletal muscle, adipose tissue, and brain (Correia et al. 2015). Interestingly, the PGC-1 $\alpha$ gene can be transcribed from alternative promoters and its transcripts can be spliced to generate several PGC- $1 \alpha$ variants with different biological activities (Martínez-Redondo et al. 2015; Ruas et al. 2012). The expression of PGC- $1 \alpha 1$ [the founding member of the family (Puigserver et al. 1998)] is increased by aerobic exercise and regulates genes involved in mitochondrial biogenesis, adaptive thermogenesis, lipid and glucose homeostasis, and fiber-type switching, among others (Correia et al. 2015). For these reasons, reduced PGC- $1 \alpha 1$ expression in different tissues has been linked to obesity, diabetes, sarcopenia, and neurodegeneration. Conversely, it has been shown that sustained PGC-1 $\alpha 1$ expression in mouse skeletal muscle has several beneficial effects. PGC-1 $1 \alpha 4$ is induced by resistance exercise training and promotes skeletal muscle growth and strength. Importantly, transgenic animals with elevated PGC-1 $\alpha 4$ levels in skeletal muscle show increased exercise performance and resistance to 
atrophy and to cancer-induced cachexia (Ruas et al. 2012). Of the many other PGC- $1 \alpha$ isoforms (Martínez-Redondo et al. 2015), PGC-1 $\alpha 2$ and $\alpha 3$ have been shown to be involved in alternative splicing of their target genes (Martinez-Redondo et al. 2016), but their main biological roles in skeletal muscle remain unknown.

In addition to helping us understand local adaptations in skeletal muscle, PGC-1 $\alpha$ coactivators have been used as tools to investigate the distal actions of exerciseinduced myokines. This research has been greatly helped by the use of mouse genetic models with tissue-specific gain or loss of PGC-1 $\alpha$ function developed to mimic the physiological and pathophysiological situations associated with altered PGC- $1 \alpha$ expression (Rowe and Arany 2014). From these efforts, PGCs have been found to regulate the expression of several myokines involved in inflammation (Handschin and Spiegelman 2008), angiogenesis (VEGF; Arany et al. 2008; Chinsomboon et al. 2009), non-shivering thermogenesis (Fndc5/Irisin, Meteorinlike 1, and $\beta$-aminoisobutyric acid; Boström et al. 2012; Rao et al. 2014; Roberts et al. 2014), and muscle mass regulation (Myostatin; Ruas et al. 2012). Similarly, the observation that muscle-specific PGC- $1 \alpha 1$ transgenic mice (MKC-PGC-1 $\alpha$; Lin et al. 2002) are resistant to developing depressive-like behaviors when exposed to chronic mild stress resulted in the identification of skeletal muscle as an important site for kynurenine (Kyn) detoxification (Agudelo et al. 2014). Kyn and some of its metabolites are known neurotoxic compounds linked to mental health disorders, such as depression and schizophrenia (Cervenka et al. 2017).

\section{The Kyn Pathway of Tryptophan Degradation}

Tryptophan (TRP) is an essential amino acid that cannot be synthesized by the human body and must be acquired from diet. TRP is taken up through the large neutral amino acid transporter (LAT) isoforms 1 to 4 , which are also responsible for the uptake of other amino acids such as phenylalanine, tyrosine, and cysteine, and others. However, with the exception of the blood-brain barrier (BBB), LATs have sufficient capacity to avoid significant competition between TRP and other amino acids. A large fraction of plasma TRP circulates bound to albumin and is thus not available for cellular uptake and usage, since only free TRP is taken up by LATs. Under normal circumstances, only about $1 \%$ of absorbed TRP is used in protein synthesis, $4-5 \%$ is used in the production of the neurotransmitter serotonin and the hormone melatonin, and about $95 \%$ is catabolized by the Kyn pathway of TRP degradation (KP). The KP generates $\mathrm{NAD}^{+}$as an end-product (mainly in liver and kidney; Houtkooper et al. 2010) and a collection of intermediary metabolites with diverse biological activities (Fig. 1), which have been mainly studied in the context of psychiatric disease.

There are two isozymes that catalyze the first step of TRP degradation: tryptophan 2,3-dioxygenase (TDO) and indoleamine 2,3-dioxygenase (IDO). TDO is specific for TRP and has a more restricted expression pattern (highest in the liver; Yu et al. 2016), whereas IDO is more ubiquitous and metabolizes any compound with 


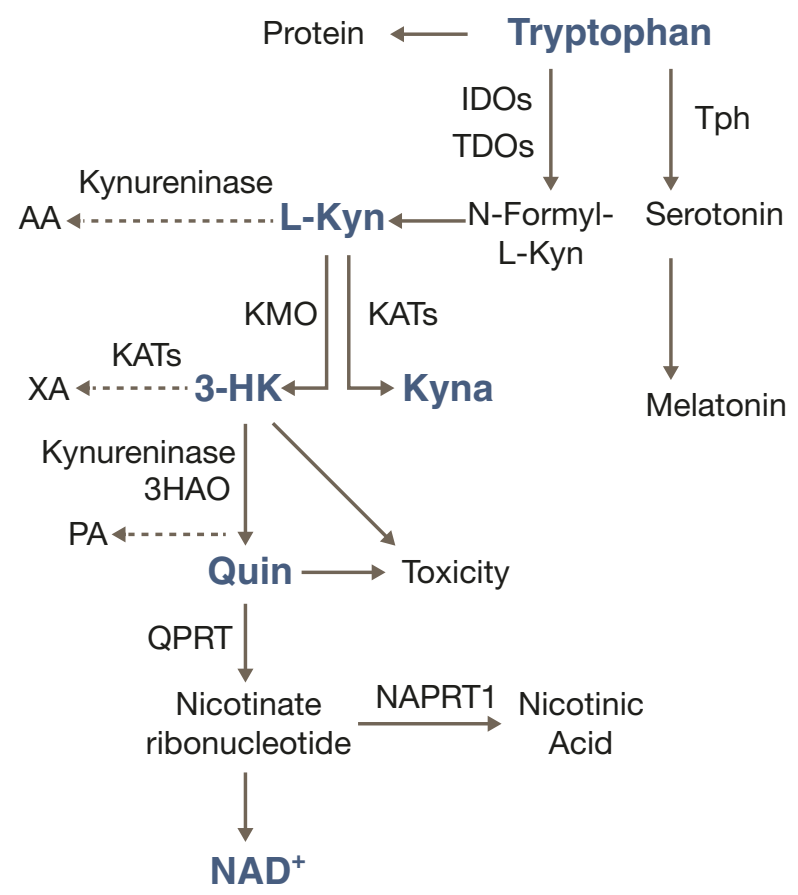

Fig. 1 The kynurenine pathway of tryptophan degradation. Tph tryptophan hydroxylase, $I D O$ indoleamine 2,3-dioxygenase, TDO tryptophan 2,3-dioxygenase, L-Kyn L-kynurenine, $A A$ Anthranilic acid, KMO Kynurenine 3-Monooxygenase, KATs Kynurenine amino-transferases, 3HAO 3-Hydroxyanthranilate 3,4-Dioxygenase, PA Picolinic acid, Quin Quinolinic acid, QPRT Quinolinate phosphoribosyltransferase, $N A D^{+}$Nicotinamide adenine dinucleotide, $N A P R T 1$ Nicotinate phosphoribosyltransferase

an indol ring structure. The expression and activity of TDO are regulated by cues that include tryptophan, glucocorticoid, and estrogen levels (Yu et al. 2016) and are inhibited under pro-inflammatory conditions. Under those conditions, liver TDO ceases to be the main isozyme responsible for TRP metabolism (normally accounting for $90 \%$ ), and the extra-hepatic IDO takes on a more prominent role. This shift in TRP metabolism causes an increase of Kyn produced by immune cells that express high levels of IDO in an attempt to control the inflammatory environment. Indeed, as pro-inflammatory cytokines stimulate IDO activity, rising Kyn levels activate the aryl hydrocarbon receptor (AhR) in discrete immune populations and reduce the activity of natural killer cells (NKT), dendritic cells (DC), and T-cells while allowing for Treg proliferation. The net result of this process is an increase in immune tolerance. In line with this, some tumor cells express high IDO/TDO levels as a strategy for escaping the immune system (Platten et al. 2015). Importantly, not all cells possess the complete enzymatic machinery to fully convert TRP to NAD+. In general, the metabolites generated by the KP depend on which enzymes are expressed in each cell type. For example, Kyn aminotransferases (KATs), of which 
there are four genes in the human genome, are responsible for metabolizing Kyn to kynurenic acid (Kyna) and 3-hydroxykynurenine (3-HK) to xanthurenic acid (XA) (Fig. 1). This is particularly important in the brain, as both Kyna or XA have neuroprotective properties as opposed to several of the downstream metabolites of the KP.

\section{KP Metabolites and Mental Health}

The link between the KP and central nervous system (CNS) toxicity has been appreciated for a long time (Lapin 1978). However, it was only in the early 1980s that quinolinic acid (QA) was shown to be a NMDAR agonist (Stone and Perkins 1981), thus providing the first mechanism for KP-induced CNS toxicity. The majority of Kyn reaches the brain from the periphery, as it can easily cross the BBB. However, Kyn can also be produced locally from TRP by astrocytes and microglial cells (Guillemin et al. 2001; Stone and Darlington 2002). Interestingly, although Kyn accumulation in the CNS is consistently associated with neuroinflammation and negative outcomes, its precise mechanism of action remains elusive. In fact, activation of astrocytic AhR (for which Kyn is an agonist) has been shown to be antiinflammatory in the context of multiple sclerosis (Rothhammer et al. 2016). To date, KP-associated toxicity is mostly understood as the balance between the excitotoxic actions of QA and the neuroprotective effect of Kyna as a NMDAR and $\alpha 7$-nicotinic acetylcholine receptor $(\alpha 7$-nAChR) antagonist. Microglial cells can produce QA whereas astrocytes preferentially generate Kyna (although they can produce QA from extracellular 3-HK, which increases with neuroinflammation). Interestingly, elevated Kyna levels have been found in the cerebrospinal fluid of schizophrenic patients (Linderholm et al. 2012). Conversely, reducing brain Kyna levels in preclinical studies (using a KAT2 inhibitor) resulted in improved cognitive function during chemically induced challenges to working and spatial memory tasks (Kozak et al. 2014). In this situation, the pathological consequences of high Kyna concentrations are likely to manifest due to hypoglutamatergic function. 3-HK induces neuronal apoptosis through free-radical generation (Okuda et al. 1998; Polyzos and Ketelhuth 2015). Several metabolites of the pathway have also been linked to neurodegenerative diseases such as Alzheimer's disease, Huntington's disease, Parkinson's disease, among others.

\section{Crosstalk Between Physical Exercise and KP Metabolites}

The importance of TRP in exercise has been extensively investigated (Newsholme and Blomstrand 2006), not only due to its critical role in protein synthesis but also in efforts to understand the development of central fatigue and the interplay between diet, exercise, and mood disorders. For example, the increase in circulating free fatty acids that occurs during endurance exercise displaces TRP from albumin and 
increases free TRP levels, resulting in higher TRP uptake and metabolism in different tissues, with direct impact on the KP. Accordingly, an increase in free TRP plasma levels is followed by an increase in TRP and some of its metabolites in several brain regions associated with the control of mood and fatigue (Blomstrand et al. 1989; Newsholme and Blomstrand 2006).

High TRP uptake during exercise could lead to higher $\mathrm{NAD}^{+}$production, which is indeed increased in tissues such as the liver (which is able to fully catabolize TRP). However, the risk of accumulating KP metabolites that can cross the BBB (e.g., Kyn and 3-HK) could have detrimental effects. Recently, it has been shown that exercise increases the expression of KAT enzymes in skeletal muscle (Agudelo et al. 2014; Schlittler et al. 2016), promoting the conversion of Kyn into Kyna, which does not cross the BBB (Agudelo et al. 2014). Peripheral Kyn catabolism prevents its accumulation in the brain and the associated deleterious effects. This is particularly relevant in the context of stress-induced depression, which is characterized by high Kyn levels. This work has provided a mechanism for how physical exercise can improve mental health. Exercise training activates muscle KAT expression and Kyn detoxification by inducing the expression of PGC- $1 \alpha 1$ and the PPAR $\alpha / \delta$ transcription factors, which offers potential therapeutic targets for future antidepressant medications. Interestingly, elevated $\mathrm{NAD}^{+}$levels could further promote this mechanism, as NAD+ is a positive regulator of Sirtuin1, which in turn activates PGC-1 $\alpha 1$ through lysine deacetylation (Rodgers et al. 2005). Sirtuins have multiple effects on the regulation of cellular energy metabolism, metabolic enzymes and oxidative stress responses in mitochondria (Cantó et al. 2015). Therefore, alterations in the KP may ultimately affect skeletal muscle metabolism and function through multiple concurrent mechanisms.

\section{Future Perspectives and Considerations}

TRP is without doubt an important player in many critical functions of a living organism. In addition to being a building block for protein synthesis, TRP plays a role in mental health, energy homeostasis, and immune regulation. It is exciting to see research in these different areas becoming more and more interdisciplinary.

Although we have focused here mainly on post-absorptive TRP metabolism, this amino acid can be sequestered by gut microbiota and converted to tryptamine (Williams et al. 2014) or other indole compounds such as indole-3-propionic acid and indole-3-aldehyde (Rothhammer et al. 2016). Indol compounds serve as important signals between bacteria and between bacteria and their host. Indols are also AhR agonists involved in the local immunomodulation of host tolerance responses and, distally, in the regulation of CNS inflammation (Hubbard et al. 2015). It is therefore critical to consider the effect of the microbiome on TRP metabolism and host physiology. For example, germ-free (GF) mice show reduced anxiety-like behavior, and this phenotype is reversed after colonization. This observation suggests that gut-derived molecules can modulate mood and possibly cognition, and it 
opens interesting challenges in the discovery of novel pathways of gut-brain communication (Heijtz et al. 2011). Physical exercise also has an impact on gut microbiome composition, but it remains to be explored if this directly impacts on gut TRP metabolism.

The KP is highly conserved evolutionarily, which allows for its study in simpler organisms to dissect its many functions. For example, Kyna has been shown to be involved in $C$. elegans feeding behavior in a NMDAR-dependent mechanism (Lemieux et al. 2015). It will be interesting to continue exploring the role of KP metabolites as possible mediators of inter-organ communication, which could integrate nutrition, metabolism, and immune responses, with all the physiological and pathophysiological implications this could have.

Acknowledgments The authors wish to acknowledge members of the Ruas lab for critical reading of the manuscript and funding from the Swedish Research Council, the Novo Nordisk Foundation (Denmark), Karolinska Institutet, The Lars Hierta Memorial Foundation, The Strategic Research Program (SRP) in Diabetes, and The SRP in Regenerative Medicine at Karolinska Institutet.

\section{References}

Agudelo LZ, Femenía T, Orhan F, Porsmyr-Palmertz M, Goiny M, Martinez-Redondo V, Correia JC, Izadi M, Bhat M, Schuppe-Koistinen I, Pettersson AT, Ferreira DM, Krook A, Barres R, Zierath JR, Erhardt S, Lindskog M, Ruas JL (2014) Skeletal muscle PGC-1 11 modulates kynurenine metabolism and mediates resilience to stress-induced depression. Cell 159:33-45

Arany Z, Foo S-Y, Ma Y, Ruas JL, Bommi-Reddy A, Girnun G, Cooper M, Laznik D, Chinsomboon J, Rangwala SM, Baek KH, Rosenzweig A, Spiegelman BM (2008) HIF-independent regulation ofVEGF and angiogenesis by the transcriptional coactivator PGC-1a. Nat Lett 451:1008-1013

Blomstrand E, Perrett D, Parry-Billings M, Newsholme E (1989) Effect of sustained exercise on plasma amino acid concentrations and on 5-hydroxytryptamine metabolism in six different brain regions in the rat. Acta Physiol Scand 136:473-481

Boström P, Wu J, Jedrychowski MP, Korde A, Ye L, Lo JC, Rasbach KA, Boström EA, Choi JH, Long JZ, Kajimura S, Zingaretti MC, Vind BF, Tu H, Cinti S, Højlund K, Gygi SP, Spiegelman BM (2012) A PGC1- $\alpha$-dependent myokine that drives brown-fat-like development of white fat and thermogenesis. Nature 48:463-468

Cantó C, Menzies KJ, Auwerx J (2015) NAD+ metabolism and the control of energy homeostasis: a balancing act between mitochondria and the nucleus. Cell Metab 22:31-53

Cervenka I, Agudelo LZ, Ruas JL (2017) Kynurenines: tryptophan's fantastic metabolites in exercise, inflammation, and mental health. Science (in press)

Chinsomboon J, Ruas J, Gupta RK, Thom R, Shoag J, Rowe GC, Sawada N, Raghuram S, Arany $\mathrm{Z}$ (2009) The transcriptional coactivator PGC- $1 \alpha$ mediates exercise-induced angiogenesis in skeletal muscle. Proc Natl Acad Sci 106:21401-21406

Cooper C, Moon HY, van Praag H (2017) On the run for hippocampal plasticity. Cold Spring Harb Perspect Med a029736

Correia JC, Ferreira DMS, Ruas JL (2015) Intercellular: local and systemic actions of skeletal muscle PGC-1s. Trends Endocrinol Metab 26:305-314

Giudice J, Taylor JM (2017) Muscle as a paracrine and endocrine organ. Curr Opin Pharmacol 34:49-55 
Guillemin GJ, Kerr SJ, Smythe GA, Smith DGN, Kapoor V, Armati PJ, Croitoru J, Brew BJ (2001) Kynurenine pathway metabolism in human astrocytes: a paradox for neuronal protection. J Neurochem 78:842-853

Handschin C, Spiegelman BM (2008) The role of exercise and PGC1 $\alpha$ in inflammation and chronic disease. Nature 454:463-469

Hawley JA (2004) Exercise as a therapeutic intervention for the prevention and treatment of insulin resistance. Diabetes Metab Res Rev 20:383-393

Hawley JA, Hargreaves M, Joyner MJ, Zierath JR (2014) Integrative biology of exercise. Cell 159:738-749

Heijtz RD, Wang S, Anuar F, Qian Y, Bjorkholm B, Samuelsson A, Hibberd ML, Forssberg H, Pettersson S (2011) Normal gut microbiota modulates brain development and behavior. Proc Natl Acad Sci 108:3047-3052

Houtkooper RH, Canto C, Wanders RJ, Auwerx J (2010) The secret life of NAD+: an old metabolite controlling new metabolic signaling pathways. Endocr Rev 31:194-223

Hubbard TD, Murray IA, Perdew GH (2015) Indole and tryptophan metabolism: endogenous and dietary routes to Ah receptor activation. Drug Metab Dispos 43:1522-1535

Kozak R, Campbell BM, Strick CA, Horner W, Hoffmann WE, Kiss T, Chapin DS, McGinnis D, Abbott AL, Roberts BM, Fonseca K, Guanowsky V, Young DA, Seymour PA, Dounay A, Hajos M, Williams GV, Castner SA (2014) Reduction of brain kynurenic acid improves cognitive function. J Neurosci 34:10592-10602

Lapin IP (1978) Stimulant and convulsive effects of kynurenines injected into brain ventricles in mice. J Neural Transm 42:37-43

Lemieux GA, Cunningham KA, Lin L, Mayer F, Werb Z, Ashrafi K (2015) Kynurenic acid is a nutritional cue that enables behavioral plasticity. Cell 160:119-131

Lin J, Wu H, Tarr PT, Zhang C-Y, Wu Z, Boss O, Michael LF, Puigserver P, Isotani E, Olson EN, Lowell BB, Bassel-Duby R, Spiegelman BM (2002) Transcriptional co-activator PGC-1 a drives the formation of slow-twitch muscle fibre. Nature 418:797-801

Linderholm KR, Skogh E, Olsson SK, Dahl ML, Holtze M, Engberg G, Samuelsson M, Erhardt S (2012) Increased levels of kynurenine and kynurenic acid in the CSF of patients with schizophrenia. Schizophr Bull 38:426-432

Martínez-Redondo V, Pettersson AT, Ruas JL (2015) The hitchhiker's guide to PGC-1a isoform structure and biological functions. Diabetologia 58:1969-1977

Martinez-Redondo V, Jannig PR, Correia JC, Ferreira DMS, Cervenka I, Lindvall JM, Sinha I, Izadi M, Pettersson-Klein AT, Agudelo LZ, Gimenez-Cassina A, Brum PC, Dahlman-Wright K, Ruas J (2016) Peroxisome proliferator-activated receptor gamma coactivator-1a isoforms selectively regulate multiple splicing events on target genes. J Biol Chem 291:15169-15184

Newsholme EA, Blomstrand E (2006) Branched-chain amino acids and central fatigue. J Nutr 136:274-276

Okuda S, Nishiyama N, Saito H, Katsuki H (1998) 3-Hydroxykynurenine, an endogenous oxidative stress generator, causes neuronal cell death with apoptotic features and region selectivity. J Neurochem 70:299-307

Platten M, von Knebel Doeberitz N, Oezen I, Wick W, Ochs K (2015) Cancer immunotherapy by targeting IDO1/TDO and their downstream effectors. Front Immunol 6:1-7

Polyzos KA, Ketelhuth DFJ (2015) The role of the kynurenine pathway of tryptophan metabolism in cardiovascular disease. Hamostaseologie 35:128-136

Puigserver P, Wu Z, Park CW, Graves R, Wright M, Spiegelman BM (1998) A cold-inducible coactivator of nuclear receptors linked to adaptive thermogenesis. Cell 92:829-839

Rao RR, Long JZ, White JP, Svensson KJ, Lou J, Lokurkar I, Jedrychowski MP, Ruas JL, Wrann CD, Lo JC, Camera DM, Lachey J, Gygi S, Seehra J, Hawley JA, Spiegelman BM (2014) Meteorin-like is a hormone that regulates immune-adipose Iinteractions to increase beige fat thermogenesis. Cell 157:1279-1291

Roberts LD, Bostrom P, O'Sullivan JF, Schinzel RT, Lewis GD, Dejam A, Lee Y-K, Palma MJ, Calhoun S, Georgiadi A, Chen MH, Ramachandran VS, Larson MG, Bouchard C, Rankinen T, 
Souza AL, Clish CB, Wang TJ, Estall JL, Soukas AA, Cowan CA, Spiegelman BM, Gerszten RE (2014) Beta-aminoisobutyric acid induces browning of white fat and hepatic beta-oxidation and is inversely correlated with cardiometabolic risk factors. Cell Metab 19:96-108

Rodgers JT, Lerin C, Haas W, Gygi SP, Spiegelman BM, Puigserver P (2005) Nutrient control of glucose homeostasis through a complex of PGC-1a and SIRT1. Nature 434:113-118

Rothhammer V, Mascanfroni ID, Bunse L, Takenaka MC, Kenison JE, Mayo L, Chao C-C, Patel B, Yan R, Blain M, Alvarez JI, Kébir H, Anandasabapathy N, Izquierdo G, Jung S, Obholzer N, Pochet N, Clish CB, Prinz M, Prat A, Antel J, Quintana FJ (2016) Type I interferons and microbial metabolites of tryptophan modulate astrocyte activity and central nervous system inflammation via the aryl hydrocarbon receptor. Nat Med 22:586-597

Rowe GC, Arany Z (2014) Genetic models of PGC-1 and glucose metabolism and homeostasis. Rev Endocr Metab Disord 15:21-29

Ruas JL, White JP, Rao RR, Kleiner S, Brannan KT, Harrison BC, Greene NP, Wu J, Estall JL, Irving BA, Lanza IR, Rasbach KA, Okutsu M, Nair KS, Yan Z, Leinwand LA, Spiegelman BM (2012) A PGC-1 $\alpha$ isoform induced by resistance training regulates skeletal muscle hypertrophy. Cell 151:1319-1331

Schlittler M, Goiny M, Agudelo LZ, Venckunas T, Brazaitis M, Skurvydas A, Kamandulis S, Ruas JL, Erhardt S, Westerblad H, Andersson DC (2016) Endurance exercise increases skeletal muscle kynurenine aminotransferases and plasma kynurenic acid in humans. Am J Physiol Cell Physiol 310:C836-C840

Stone TW, Darlington LG (2002) Endogenous kynurenines as targets for drug discovery and development. Nat Rev Drug Discov 1:609-620

Stone TW, Perkins MN (1981) Quinolinic acid: a potent endogenous excitant at amino acid receptors in CNS. Eur J Pharmacol 72:411-412

Williams BB, Van Benschoten AH, Cimermancic P, Donia MS, Zimmermann M, Taketani M, Ishihara A, Kashyap PC, Fraser JS, Fischbach MA (2014) Discovery and characterization of gut microbiota decarboxylases that can produce the neurotransmitter tryptamine. Cell Host Microbe 16:495-503

Yu CP, Pan ZZ, Luo DY (2016) TDO as a therapeutic target in brain diseases. Metab Brain Dis 31:737-747

Open Access This chapter is licensed under the terms of the Creative Commons Attribution 4.0 International License (http://creativecommons.org/licenses/by/4.0/), which permits use, sharing, adaptation, distribution and reproduction in any medium or format, as long as you give appropriate credit to the original author(s) and the source, provide a link to the Creative Commons license and indicate if changes were made.

The images or other third party material in this chapter are included in the chapter's Creative Commons license, unless indicated otherwise in a credit line to the material. If material is not included in the chapter's Creative Commons license and your intended use is not permitted by statutory regulation or exceeds the permitted use, you will need to obtain permission directly from the copyright holder.

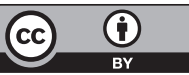

